\title{
Draft Genome of the Macadamia Husk Spot Pathogen, Pseudocercospora macadamiae
}

\author{
Olufemi A. Akinsanmi ${ }^{\dagger}$ and Lilia C. Carvalhais
}

The University of Queensland, Centre for Horticultural Science, Queensland Alliance for Agriculture and Food Innovation, Ecosciences Precinct, GPO 267, Brisbane 4001, Queensland, Australia

\begin{abstract}
Pseudocercospora macadamiae causes husk spot in macadamia in Australia. Lack of genomic resources for this pathogen has restricted acquiring knowledge on the mechanism of disease development, spread, and its role in fruit abscission. To address this gap, we sequenced the genome of $P$. macadamiae. The sequence was de novo assembled into a draft genome of 40 $\mathrm{Mb}$, which is comparable to closely related species in the family Mycosphaerellaceae. The draft genome comprises 212 scaffolds, of which 99 scaffolds are over $50 \mathrm{~kb}$. The genome has a $49 \%$ GC content and is predicted to contain 15,430 protein-coding genes. This draft genome sequence is the first for $P$. macadamiae and represents a valuable resource for understanding genome evolution and plant disease resistance.
\end{abstract}

\section{Genome Announcement}

Macadamia is the most economically important tree nut crop in the family Proteaceae (Nock et al. 2014). Macadamias are cultivated for their edible cream-colored nut (kernel), which is enclosed in a very hard shell and husk (pericarp). The four species in the genus Macadamia (M. integrifolia, M. tetraphylla, M. jansenii, and M. ternifolia) originate from the tropical forest fringes of coastal southeast Queensland and northeast New South Wales in Australia (Gross and Weston 1992). M. integrifolia, M. tetraphylla, and their hybrids are cultivated in commercial orchards in several tropical and frost-free subtropical regions in Australia, South Africa, Kenya, Brazil, Hawaii, China, Vietnam, Guatemala, Mexico, Columbia, and Malawi. In Australia, Pseudocercospora macadamiae infects the macadamia fruit pericarp causing husk spot (Beilharz et al. 2003). Husk spot is a serious disease of macadamia, resulting in extensive premature fruit abscission, giving rise to nuts with low oil content (Akinsanmi et al. 2007; Beilharz et al. 2003). Infection occurring during the early phase of fruit development may have an incubation period up to 3 months before symptoms are expressed (Akinsanmi et al. 2007, 2016; Miles et al. 2010). Moreover, the biomass of $P$. macadamiae in infected tissue increases with symptom development (Ong et al. 2016). Husk spot symptoms appear in the fruit pericarp at near fruit maturity as chlorotic flecks and progress to necrotic lesions (Miles et al. 2010; Miles et al. 2009). Fruit abscission due to husk spot commences from the chlorotic-symptom stage (Akinsanmi et al. 2016); however, the mechanisms that trigger husk spot symptom expression and fruit abscission of diseased fruit are not well understood. In macadamia, high levels of abscisic acid (ABA) were associated with period of abscission of immature fruit and $P$. macadamiae was shown to produce ABA in vitro (McConchie and Salter 2005). Therefore, P. macadamiae possibly contributes to the induction of the fruit abscission process in macadamia (Akinsanmi et al. 2016; McConchie and Salter 2005).

${ }^{\dagger}$ Corresponding author: O. A. Akinsanmi; o.akinsanmi@uq.edu.au

The author(s) declare no conflict of interest.

Accepted for publication 21 April 2020.

\section{Funding}

This research was funded by Hort Innovation using the macadamia research and development levy and funds from the Australian Government project number MC16018.

\section{Keywords}

analytical plant pathology, mycology, whole genome sequencing 
Table 1. Metrics of the draft genome of Pseudocercospora macadamiae (isolate BRIP 55526)

\begin{tabular}{lc} 
Features & Statistics \\
Estimated genome size (bp) & $40,070,143$ \\
Number of scaffolds $\geq 2 \mathrm{~kb}$ & 212 \\
L50 scaffold (bp) & 608,373 \\
N50 scaffolds & 19 \\
Maximum scaffold length (bp) & $3,067,473$ \\
Maximum contig length (bp) & $3,067,473$ \\
Number of scaffolds >50 kb & 99 \\
GC content (\%) & $49 \%$ \\
Predicted protein-coding genes & 15,430 \\
Median protein length & 321 \\
Mean protein length & 463.32 \\
Complete BUSCOs (C) & 1,287 \\
Complete and single-copy BUSCOs (S) & 1,285 \\
Complete and duplicated BUSCOs (D) & 2 \\
Fragmented BUSCOs (F) & 5 \\
Missing BUSCOs (M) & 23 \\
Total BUSCO groups searched & 1,315 \\
\hline
\end{tabular}

Phylogenetic analysis of the 28S ribosomal RNA gene revealed that $P$. macadamiae falls within the Pseudocercospora genus complex (Ong et al. 2016). The Pseudocercospora genus complex accommodates most Pseudocercospora species, representing a wide range of host plant species, including those species of Pseudocercospora with publicly available genomes and known metabolic profiles including $P$. fijiensis (previously $M$. fijiensis), the causal agent of black Sigatoka disease of banana (Crous et al. 2012). Like P. fijiensis, and many other hemibiotrophic fungal pathogens, $P$. macadamiae may produce metabolites and proteins that kill the host tissue, which could influence the virulence and adaptation of the pathogen (Chang et al. 2016; Noar and Daub 2016). However, there is no information on the repertoire of effectors and toxic metabolites produced by $P$. macadamiae during host infection and disease development.

In this study, we used a holotype isolate of $P$. macadamiae (BRIP 55526) that was collected from a husk spot lesion of the macadamia cultivar $\mathrm{H} 2$ (Hinde) obtained from a commercial orchard in the Glasshouse Mountains, Queensland, Australia. A monoconidial culture of the isolate was grown on half-strength potato dextrose agar (Difco Laboratories Inc., Sparks, MD) and the genomic DNA was extracted as described by Ong et al. (2016). Libraries were prepared according to the manufacturer's protocol using a Nextera DNA Flex Library Preparation Kit (20018705, Illumina, San Diego, CA). The library was prepared for sequencing on a NextSeq500 (Illumina) using NextSeq 500/550 High Output v2 $2 \times 150$ bp paired-end chemistry at the Australian Centre for Ecogenomics according to the manufacturer's protocol. Raw pairedend reads were quality-checked and low-quality reads and adaptors were removed using Trimmomatic v0.36 (Bolger et al. 2014). Contigs with lengths less than 2,000 bp were removed using BBMap (ver. 36.92) (Bushnell 2014) and the quality of the genome assembly was calculated using the Benchmarking Universal Single-Copy Orthologs tool (BUSCO v3.02) (Simão et al. 2015) with the Ascomycota lineage dataset. De novo assembly was performed using Spades v3.13 (Bankevich et al. 2012) from both the paired-end reads and resulted in 212 scaffolds with an average size of $608,373 \mathrm{bp}$. Ninety-nine scaffolds were over $50 \mathrm{~kb}$. The wholegenome assembly resulted in a draft genome with an overall GC content of $49 \%$, and the full genome size is estimated to be $40.0 \mathrm{Mb}$. Statistics of genome sequencing and assembly are summarized (Table 1). Most hits from the BLAST analysis correspond to sequences of the genera Pseudocercospora and Passalora. RepeatMasker v4.0.8 (Tarailo-Graovac and Chen 2009) was used to identify and mask repeat regions and GeneMark v4.38 (Besemer and Borodovsky 2005) was used to predict protein-coding genes within the masked scaffolds using the self-training and fungus algorithm options. Functional annotation of protein sequences was conducted using BLASTP within DIAMOND v0.9.22 (Buchfink et al. 2015). Protein sequences were aligned against Uniref100 (release 2018-3) (UniProt Consortium 2010) with an associated KEGG ID (Kanehisa et al. 2007) and Swissprot (release 2019-01) (Boeckmann et al. 2003) databases. In addition, eggNOG-mapper (Huerta-Cepas et al. 2017) was used to improve the functional annotation of the predicted proteins and 7,415 were assigned to orthologous group.

Gene prediction analysis yielded a total of 15,430 protein-coding genes. To calculate the average amino acid identity (AAl) between the predicted proteome and the species present in the UniProt database (UniProt Consortium 2017), we ran the predicted proteins through the 
software AAI profiler (Medlar et al. 2018). The analysis revealed the three organisms with the greatest similarity to $P$. macadamiae were $P$. fijiensis $(\mathrm{AAI}=81 \%), P$. eumusae $(\mathrm{AAI}=80 \%)$, and $P$. musae $(\mathrm{AAI}=80 \%)$. RepeatMasker identified 6,120 repeats in the $P$. macadamiae genome, which included 5,643 simple repeats and 477 low complexity repeats, and TransposonPSI (http://transposonpsi.sourceforge.net) predicted 3,604 transposable elements that belonged to various transposon classes. The genome annotation completeness was assessed using BUSCO (Simão et al. 2015), which identified 1,285 complete benchmarking universal singlecopy orthologs (BUSCOs) in the database of ascomycetes. SignalP v5.0 (Armenteros et al. 2019) predicted 389 secreted proteins, and EffectorP 2.0 (Sperschneider et al. 2018) predicted 66 of these as effectors. In addition, TMHMM 2.0 (Krogh et al. 2001) predicted that 2,955 of $15,430(19.2 \%$ of all proteins) are transmembrane helices and out of the 66 proteins that were predicted as effectors, four were predicted to be transmembrane helices. The potential of $P$. macadamiae to degrade and metabolize a range of polysaccharides was evaluated with the automated carbohydrate active enzymes (CAZymes) annotation software dbCAN (Zhang et al. 2018), which predicted 676 putative CAZymes (HMMER, E-value < 1e-15, coverage $>0.35$ ). Of the six major superfamilies, glycoside hydrolases $(\mathrm{GH})$ is the most prevalent, representing $45.6 \%$ of the total predicted CAZyome, followed by auxiliary activities (18.9\%), glycosyltransferases (16.4\%), carbohydrate esterase (15.7\%), carbohydrate-binding module $(2.1 \%)$, and polysaccharide lyases (1.3\%). Similar percentages of $\mathrm{GH}$ was found in the CAZyome of other closely related Pseudocercospora species, P. fijiensis (47.2\%), P. musae (45.3\%), and $P$. eumusae (47.1\%) (Chang et al. 2016). The genome sequence of $P$. macadamiae is an important resource for future comparative genomic analyses with other Pseudocercospora species and useful for improved understanding of the pathogen biology, epidemiology, and host-pathogen interaction.

Accession number. The assembled genome sequence of $P$. macadamiae isolate BRIP 55526 has been deposited in GenBank under the accession number WRNY00000000. The data for this experiment have been submitted to NCBI with SRA submission SUB6642804.

\section{Literature Cited}

Akinsanmi, O. A., Miles, A. K., and Drenth, A. 2007. Timing of fungicide application for control of husk spot caused by Pseudocercospora macadamiae in macadamia. Plant Dis. 91:1675-1681.

Akinsanmi, O. A., Miles, A. K., and Drenth, A. 2016. Fruit abscission in macadamia due to husk spot disease. Acta Hortic. 1109:209-214.

Armenteros, J. J. A., Tsirigos, K. D., Sønderby, C. K., Petersen, T. N., Winther, O., Brunak, S., von Heijne, G., and Nielsen, H. 2019. SignalP 5.0 improves signal peptide predictions using deep neural networks. Nat. Biotechnol. 37:420-423.

Bankevich, A., Nurk, S., Antipov, D., Gurevich, A. A., Dvorkin, M., Kulikov, A. S., Lesin, V. M., Nikolenko, S. I., Pham, S., Prijbelski, A. D., and Pyshkin, A. V. 2012. SPAdes: A new genome assembly algorithm and its applications to single-cell sequencing. J. Comput. Biol. 19:455-477.

Beilharz, V., Mayers, P. E., and Pascoe, I. G. 2003. Pseudocercospora macadamiae sp. nov., the cause of husk spot of macadamia. Australas. Plant Pathol. 32: 279-282.

Besemer, J., and Borodovsky, M. 2005. GeneMark: Web software for gene finding in prokaryotes, eukaryotes and viruses. Nucleic Acids Res.: W451-W454.

Boeckmann, B., Bairoch, A., Apweiler, R., Blatter, M. C., Estreicher, A., Gasteiger, E., Martin, M. J., Michoud, K., O'Donovan, C., Phan, I., and Pilbout, S. 2003. The SWISS-PROT protein knowledgebase and its supplement TrEMBL in 2003. Nucleic Acids Res. 31:365-370.

Bolger, A. M., Lohse, M., and Usadel, B. 2014. Trimmomatic: A flexible trimmer for Illumina sequence data. Bioinformatics 30:2114-2120.

Buchfink, B., Xie, C., and Huson, D. H. 2015. Fast and sensitive protein alignment using DIAMOND. Nat. Methods 12:59-60.

Bushnell, B. 2014. BBMap: A fast, accurate, splice-aware aligner (No. LBNL-7065E). Lawrence Berkeley National Lab (LBNL), Berkeley, CA.

Chang, T.-C., Salvucci, A., Crous, P. W., and Stergiopoulos, I. 2016. Comparative genomics of the sigatoka disease complex on banana suggests a link between parallel evolutionary changes in Pseudocercospora fijiensis and Pseudocercospora eumusae and increased virulence on the banana host. Plos Genet 12:e1005904-e.
Crous, P. W., Braun, U., Hunter, G. C., Wingfield, M. J., Verkley, G. J. M., Shin, H.-D., Nakashima, C., and Groenewald, J. Z. 2012. Phylogenetic lineages in Pseudocercospora. Stud. Mycol. 75:37-114.

Gross, C. L., and Weston, P. H. 1992. Macadamia jansenii (Proteaceae), a new species from central Queensland. Aust. Syst. Bot. 5:725-728.

Huerta-Cepas, J., Forslund, K., Coelho, L. P., Szklarczyk, D., Jensen, L. J., von Mering, C., and Bork, P. 2017. Fast genome-wide functional annotation through orthology assignment by eggNOG-Mapper. Mol. Biol. Evol. 34:2115-2122.

Kanehisa, M., Araki, M., Goto, S., Hattori, M., Hirakawa, M., Itoh, M., Katayama, T., Kawashima, S., Okuda, S., Tokimatsu, T., and Yamanishi, Y. 2007. KEGG for linking genomes to life and the environment. Nucleic Acids Res. 36:D480-D484.

Krogh, A., Larsson, B., von Heijne, G., and Sonnhammer, E. L. L. 2001. Predicting transmembrane protein topology with a hidden Markov model: Application to complete genomes. J. Mol. Biol. 305:567-580.

McConchie, C. A., and Salter, B. 2005. Investigation of Nut Abscission and Tree Shaking in Macadamia. Horticulture Australia Limited, Sydney.

Medlar, A. J., Toronen, P., and Holm, L. 2018. AAl-profiler: Fast proteome-wide exploratory analysis reveals taxonomic identity, misclassification and contamination. Nucleic Acids Res. 46:W479-W485.

Miles, A. K., Akinsanmi, O. A., Aitken, E. A. B., and Drenth, A. 2010. Timing of infection of macadamia fruit by Pseudocercospora macadamiae and climatic effects on growth and spore germination. Australas. Plant Pathol. 39:453-462.

Miles, A. K., Akinsanmi, O. A., Sutherland, P. W., Aitken, E. A. B., and Drenth, A. 2009. Infection, colonisation and sporulation by Pseudocercospora macadamiae on macadamia fruit. Australas. Plant Pathol. 38:36-43.

Noar, R. D., and Daub, M. E. 2016. Transcriptome sequencing of Mycosphaerella fijiensis during association with Musa acuminata reveals candidate pathogenicity genes. BMC Genomics 17:690.

Nock, C. J., Elphinstone, M. S., Ablett, G., Kawamata, A., Hancock, W., Hardner, C. M., and King, G. J. 2014. Whole genome shotgun sequences for microsatellite discovery and application in cultivated and wild Macadamia (Proteaceae). Appl. Plant Sci. 2:1300089. 
Ong, C. E., Henderson, J., and Akinsanmi, O. A. 2016. Characterization and development of qPCR for early detection and quantification of Pseudocercospora macadamiae at different stages of infection process. Eur. J. Plant Pathol. 147:85-102.

Simão, F. A., Waterhouse, R. M., loannidis, P., Kriventseva, E. V., and Zdobnov, E. M. 2015. BUSCO: Assessing genome assembly and annotation completeness with single-copy orthologs. Bioinformatics 31:3210-3212.

Sperschneider, J., Dodds, P. N., Gardiner, D. M., Singh, K. B., and Taylor, J. M. 2018. Improved prediction of fungal effector proteins from secretomes with EffectorP 2.0. Mol. Plant Pathol. 19:2094-2110.
Tarailo-Graovac, M., and Chen, N. 2009. Using RepeatMasker to identify repetitive elements in genomic sequences. Curr. Protoc. Bioinformatics 25:4-10.

UniProt Consortium. 2010. The universal protein resource (UniProt) in 2010. Nucleic Acids Res. 38:D142-D148.

UniProt Consortium. 2017. UniProt: The universal protein knowledgebase. Nucleic Acids Res. 45:D158-D169.

Zhang, H., Yohe, T., Huang, L., Entwistle, S., Wu, P., Yang, Z., Busk, P. K., Xu, Y., and Yin, Y. 2018. dbCAN2: A meta server for automated carbohydrate-active enzyme annotation. Nucleic Acids Res. 46:W95-W101. 